\title{
The parallel intensionally fully abstract games model of PCF
}

\author{
Simon Castellan and Pierre Clairambault \\ ENS de Lyon, CNRS, Inria, UCBL, Université de Lyon \\ LIP, 46 allée d'Italie, 69364 Lyon, France
}

\author{
Glynn Winskel \\ Computer Laboratory, University of Cambridge \\ 15 JJ Thomson Avenue, Cambridge CB3 OFD, UK
}

\begin{abstract}
We describe a framework for truly concurrent game semantics of programming languages, based on Rideau and Winskel's concurrent games on event structures. The model supports a notion of innocent strategies that permits concurrent and non-deterministic behaviour, but which coincides with traditional Hyland-Ong innocent strategies if one restricts to the deterministic sequential case. In this framework we give an alternative interpretation of Plotkin's $\mathrm{PCF}$, that takes advantage of the concurrent nature of strategies and formalizes the idea that although $\mathrm{PCF}$ is a sequential language, certain sub-computations are independent and can be computed in a parallel fashion. We show that just as Hyland and Ong's sequential interpretation of $\mathrm{PCF}$, our parallel interpretation yields a model that is intensionally fully abstract for PCF.
\end{abstract}

\section{INTRODUCTION}

Regardless of mathematical elegance, partial order models of concurrent computation are in principle more informative than their interleaving counterparts: they avoid the state explosion problem inherent to interleavings, and retain explicit information on causality. This can be useful for instance for the purposes of error diagnostics, or security analysis. However, although we have truly concurrent models for simple process languages such as $\mathrm{CCS}$, extracting partial order models from source code remains a challenge, especially if one considers rich concurrent programming languages with complex computational features such as higher-order, state or exceptions.

In order to construct compositionally a fine-grained representation of the execution of higher-order programs, game semantics is a powerful tool. Game semantics proposes to see computation as an interaction between agents (strategies) exchanging messages, hence reducing higher-order computation to the exchange of first-order tokens. Thanks to this methodology, game semantics has not only given intensionally fully abstract models of PCF $[10,1]$ but also pushed beyond the purely functional setting and given effectively presentable fully abstract models of higher-order programming languages with rich computational features such as control or state.

Most games models for concurrent programming languages $[7,12]$, however, are based on interleavings. Several truly concurrent frameworks for game semantics have been proposed $[6,14,16,9]$, but have yet to be applied to the semantics of programming languages beyond CCS or linear logic - this is in part due to the fact that truly concurrent notions of strategies are mathematically more elaborate than their interleaved counterparts, and are more subtle to handle. Moreover, changing the basic metalanguage for game semantics means losing a whole body of work, such as the pleasing characterisation offered by Hyland-Ong (HO) games of various computational effects in terms of conditions on sequential strategies. The first contribution of this paper is a framework for truly concurrent game semantics, that comprises representations of computational features such as non-determinism and concurrency while containing as a sub-case the usual HO games - in particular we have notions of visibility and innocence, that in the sequential deterministic case coincide with standard HO innocence. We believe that this framework should prove adequate for further developments on truly concurrent games models of programming languages.

Our second contribution is an application of our framework to give a parallel intensionally fully abstract model for PCF. That might seem counter-intuitive, given the status of $\mathrm{PCF}$ as a paradigmatic sequential language. However, although all the primitives of PCF are purely sequential, that does not mean that its implementation has to be sequential. In particular, operations such as the conditional if $: \mathbb{B} \rightarrow \mathbb{B} \rightarrow \mathbb{B} \rightarrow \mathbb{B}$ could in principle be optimized by evaluating all three arguments in parallel, and returning the adequate one according to the result of the first argument. Standard game semantics of PCF specify explicitly an evaluation order, and forbids this operational reading of if. Our game semantics, while authorizing the execution as prescribed by the sequential game semantics of PCF, will make this parallel computation official and express this parallelism in a truly concurrent manner. Despite this added intensional behaviour, our conditions will be enough to guarantee that our strategies are extensional, and that their extensional collapse is the fully abstract model for PCF.

Related works. On the game-theoretic front, the present contribution was made possible by the recent developments $[16,4,21]$ initiated by Rideau and Winskel around a framework for game semantics based on event structures - our basic setting is an extension of the games with symmetry of [4]. The framework of [16] generalizes earlier approaches to deterministic truly concurrent notions of games [2, 14, 6], used in particular by Melliès to build a fully complete model of full propositional linear logic [13]. Also relevant is the work of Hirschowitz and Pous [9, 8], which gives a fully abstract model of CCS w.r.t. fair testing, based on a notion of strategies as sheaves on a category of plays.

Outline. In Section II we will present our notions of games 


$$
\begin{array}{llll}
\text { Types. } \quad A, B::= & \mathbb{B}|\mathbb{N}| A \rightarrow B \\
\text { Terms. } M, N \quad:= & x|\lambda x . M| M N|Y| \\
& & \text { tt } \mid \text { ff } \mid \text { if } M_{1} M_{2} M_{3} \mid \\
& n \mid \text { succ } M \mid \text { pred } M \mid \text { iszero } M
\end{array}
$$

Fig. 1. The language PCF

and strategies and compare them with the HO innocent wellbracketed strategies. In Section III, we present the mathematical foundations for our model, and describe a sound interpretation of PCF. Finally, in Section IV we prove a finite definability result and deduce full abstraction.

\section{Parallel PCF-Strategies}

We introduce in Figure 1 the syntax of PCF as used in this paper. In this paper $\mathbb{X}$ will range over ground types, ie. $\mathbb{B}$ or $\mathbb{N}$. The typing rules we consider are standard, except for the typing rule for if that is replaced with:

$$
\frac{\Gamma \vdash M: \mathbb{B} \quad \Gamma \vdash N_{1}: \mathbb{X} \quad \Gamma \vdash N_{2}: \mathbb{X}}{\Gamma \vdash \text { if } M N_{1} N_{2}: \mathbb{X}}
$$

Note that if $N_{1}, N_{2}$ have arbitrary type $A=A_{1} \rightarrow \cdots \rightarrow A_{n} \rightarrow$ $\mathbb{X}$, if $M N_{1} N_{2}$ can still be used and is considered syntactic sugar for $\lambda x_{1} \ldots x_{n}$. if $M\left(N_{1} x_{1} \ldots x_{n}\right)\left(N_{2} x_{1} \ldots x_{n}\right)$. Terms of PCF are executed following the standard call-byname big-step operational semantics, yielding an evaluation relation $\Downarrow$ between closed terms and values (ie. constants of ground type or $\lambda$-abstraction). As usual we write $M \Downarrow$ if $M \Downarrow v$ for some $v$. In this section, we aim to explain concretely what our model computes, and how it represents programs.

\section{A. Sequential PCF-strategies presented concretely}

$\mathrm{HO}$ game semantics formalizes the intuition that a program is a strategy having a dialogue with its execution environment. A possible dialogue on the type $\mathbb{B} \Rightarrow \mathbb{B} \Rightarrow \mathbb{B} \Rightarrow \mathbb{B}$ could be:

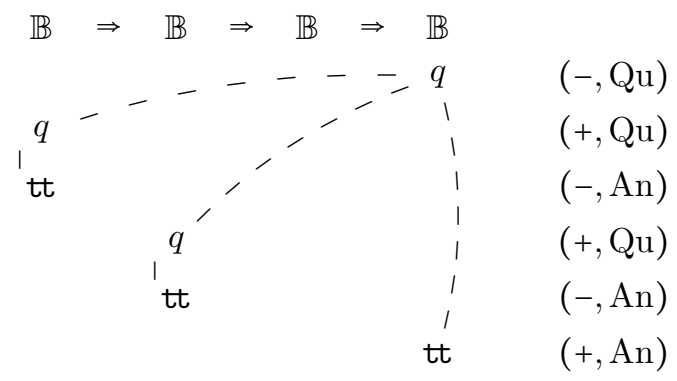

Each move is either Player or Opponent, and is either a Question or an Answer. Questions correspond to variable calls, whereas Answers indicate a call terminating. The dashed lines between moves (traditionally called justification pointers) convey information about thread indexing; in this example they are redundant but become required at higher types. Sequential innocent strategies consist of sets of dialogues as above, where Opponent moves are justified by the preceding one
- such dialogues are known as P-views. A strategy for a PCF term contains several such dialogues, specifying the term entirely. In our example, the dialogue is actually a branch of the strategy for if, that interrogates its left argument first, then the second or third depending on the result. This strategy contains in total four maximal P-views, described by:

$$
\begin{aligned}
& \mathbb{B} \Rightarrow \mathbb{B} \Rightarrow \mathbb{B} \Rightarrow \mathbb{B}
\end{aligned}
$$

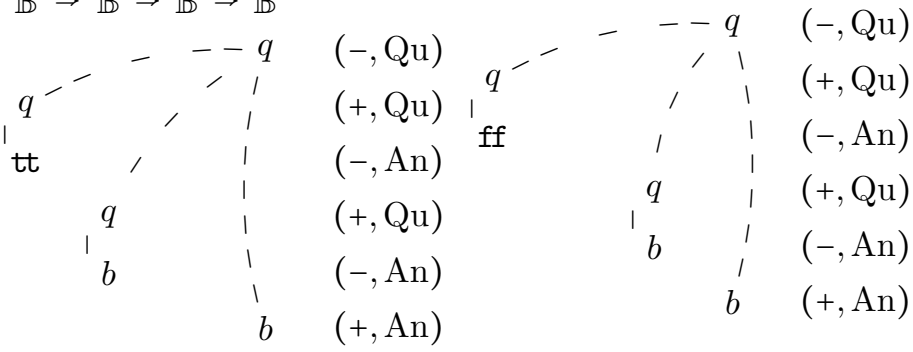

Such non-empty sets of P-views (satisfying further conditions called determinism and well-bracketing) are called sequential $\mathrm{PCF}$-strategies, or PCF-strategies for short.

\section{B. $\mathrm{PCF}_{\|}$-strategies}

Instead of investigating its arguments sequentially, an optimized implementation of if could evaluate the second and third arguments before they are needed, without waiting for the first call to terminate. This intuition leads to a different notion of strategy, and a different interpretation of PCF.

1) A partial order for if: States of this optimized strategy for if will no longer be total orders but partial orders. For instance, a state of if where the first argument returns $t t$ could be (labelling the distinct copies of $\mathbb{B}$ for clarity):

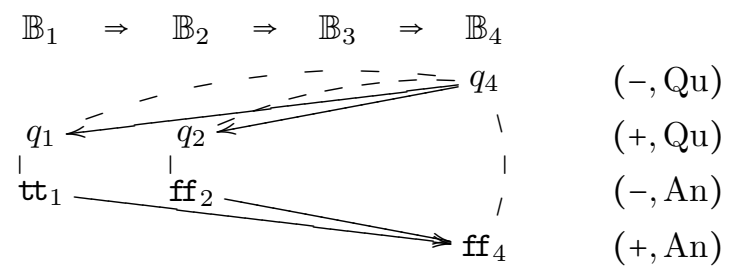

Additionally to the justification pointers, the strategy is now equipped with a relation $\rightarrow$ indicating immediate causality (omitted for readability when it coincides with vertical juxtaposition). In the sequential diagrams of Subsection II-A these were not required since in a sequential setting immediate causality is chronological contiguity. In the diagram above, any two events not related by the transitive closure of immediate causality are concurrent, and occur independently. So (amongst other actions) if interrogates its two first arguments in parallel, and is able to answer ff if the first argument returns tt and the second ff. Instead of being a total order, a "branch" of a strategy will have to be generalized to a partial order of course "branch" here is misused, instead we will speak of a prime, i.e. a partial order with a maximum element. As we will see soon, the full strategy will contain other primes for different executions leading to an answer in $\mathbb{B}_{4}$. 
2) Event structures: Even though our strategies for PCF are all deterministic, we aim for a framework that can accommodate non-deterministic behaviour. Accordingly, we will use event structures [18]: a concurrent analogue of trees, providing a description of systems that features both independence of events, and non-determinism. Formally, an event structure is $(E, \leq$, Con) with $E$ a set of events, $\leq$ a partial order indicating causal dependency, and Con a nonempty consistency relation consisting of finite subsets of $E$, such that:

$$
\begin{aligned}
& \left\{e^{\prime} \in E \mid e^{\prime} \leq e\right\} \text { is finite for all } e \in E \\
& \{e\} \in \text { Con for all } e \in E \\
& Y \subseteq X \in \text { Con } \Longrightarrow Y \in \text { Con } \\
& X \in \text { Con } \& e \leq e^{\prime} \in X \Longrightarrow X \cup\{e\} \in \text { Con }
\end{aligned}
$$

For example, the event structure for the non-deterministic strategy returning a random boolean is:

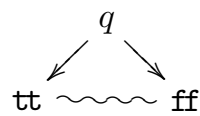

where the wiggly line indicates immediate conflict, i.e. the consistent sets are those not containing both $t t$ and ff.

Event structures will sometimes be equipped with a polarity function pol $: E \rightarrow\{-,+\}$ specifying whether an event $e$ is Player $(\operatorname{pol}(e)=+)$ or Opponent $(\operatorname{pol}(e)=-)$. An event structure $E$ with pol is called an event structure with polarities, or esp for short.

3) Notations: For $e, e^{\prime} \in E$, we write $e \rightarrow e^{\prime}$ for immediate causality, i.e. $e<e^{\prime}$ and for all $e^{\prime \prime}$ such that $e \leq e^{\prime \prime} \leq e^{\prime}$, we have $e=e^{\prime \prime}$ or $e^{\prime \prime}=e^{\prime}$. If $E$ is an event structure, we write $\mathscr{C}(E)$ for the set of configurations of $E$, comprising the finite subsets $x \subseteq_{f} E$ such that $x \in$ Con and $x$ is down-closed, i.e. for all $e \in x, e^{\prime} \leq e$, we also have $e^{\prime} \in x$. For $e \in E$, we write $[e]$ the corresponding prime configuration or prime for short, defined as $[e]=\left\{e^{\prime} \in E \mid e^{\prime} \leq e\right\}$. Finally if $E$ has polarity, we write $x \subseteq^{+} y$ (resp. $x \subseteq^{-} y$ ) if $x \subseteq y$ and $\operatorname{pol}(y \backslash x) \subseteq\{+\}$ (resp. $\operatorname{pol}(y \backslash x) \subseteq\{-\})$.

\section{Concurrent pre-strategies playing on arenas}

We now describe more formally our notion of strategies.

1) Arenas: As usual in $\mathrm{HO}$ game semantics, the types of $\mathrm{PCF}$ will be interpreted as arenas.

Definition II.1. An arena is a tuple $(A, \leq$, pol, $\lambda)$ such that $\left(A, \leq, \mathcal{P}_{f}(A), p o l\right)$ is a countable esp satisfying:

$$
\begin{aligned}
& \forall a, a^{\prime}, a^{\prime \prime} \in A, a \leq a^{\prime \prime} \wedge a^{\prime} \leq a^{\prime \prime} \Longrightarrow a \leq a^{\prime} \vee a^{\prime} \leq a \\
& \forall a, a^{\prime} \in A, a \rightarrow a^{\prime} \Longrightarrow \operatorname{pol}(a) \neq \operatorname{pol}\left(a^{\prime}\right) \\
& \forall a \in A, a \in \min (A) \Longrightarrow \operatorname{pol}(a)=-
\end{aligned}
$$

with $\min (A)$ the set of minimal events of $A$ and $\lambda: A \rightarrow$ $\{\mathrm{Qu}, \mathrm{An}\}$ a Question/Answer labeling function, such that:

$$
\begin{aligned}
& \forall a \in A, a \in \min (A) \Longrightarrow \lambda(a)=\mathrm{Qu} \\
& \forall a_{1}, a_{2} \in A, a_{1} \rightarrow a_{2} \Longrightarrow \lambda\left(a_{1}\right)=\mathrm{Qu}
\end{aligned}
$$

Readers familiar with $\mathrm{HO}$ games will recognize the notion of arenas of [10], with the partial order $\leq$ primitive rather than immediate causality $\rightarrow$ (traditionally written $\vdash$ in $\mathrm{HO}$ games).
Types of $\mathrm{PCF}_{\|}$are interpreted as arenas. In particular, the basic type $\mathbb{B}$ is interpreted as (read from top to bottom):

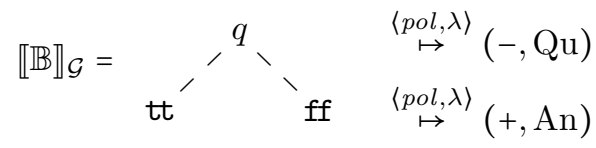

The type $\mathbb{N}$ for natural numbers is interpreted similarly, but with a countably infinite number of answers $0,1, \ldots$ In the remainder of this paper we will often omit the semantic brackets and have the same notations for constructions on types and arenas, except when brackets are useful for disambiguation.

Given two arenas $A$ and $B, A \Rightarrow B$ is defined as having:

- Events, $\{(1,(b, a)) \mid a \in A \& b \in \min (B)\} \cup\{(2, b) \mid b \in B\}$. - Causality,

$$
\begin{aligned}
& \left\{\left(\left(1,\left(b, a_{1}\right)\right),\left(1,\left(b, a_{2}\right)\right)\right) \mid a_{1} \leq a_{2} \& b \in \min (B)\right\} \cup \\
& \left\{\left(\left(2, b_{1}\right),\left(2, b_{2}\right)\right) \mid b_{1} \leq b_{2}\right\} \cup \\
& \{((2, b),(1,(b, a))) \mid a \in A \& b \in \min (B)\}
\end{aligned}
$$

- Polarity, $\operatorname{pol}((1,(b, a)))=-\operatorname{pol}(a), \operatorname{pol}((2, b))=\operatorname{pol}(b)$.

- $\mathrm{Qu} /$ An labeling, $\lambda((1,(b, a)))=\lambda(a)$ and $\lambda((2, b))=\lambda(b)$.

The reader familiar with sequential HO games will recognize here the usual arrow arena construction $A \Rightarrow B$. With these definitions, the arena $\mathbb{B} \Rightarrow \mathbb{B} \Rightarrow \mathbb{B} \Rightarrow \mathbb{B}$ of if is:

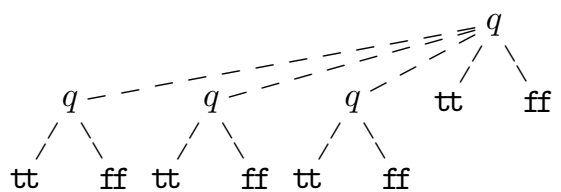

2) Expanded game: In sequential $\mathrm{HO}$ games, strategies do not play on arenas but rather on the derived game of plays with pointers [10], where the same move can be reused at will. We perform a similar construction here: from each arena we derive a game where events can be played as many times as required. Unlike sequential $\mathrm{HO}$ games though, rather than building a tree of plays we build an event structure where duplicated events retain the causal structure of the arena. Unlike sequential plays where distinct copies of the same move are kept apart chronologically, here we handle explicit copy indices.

To define it we need the notion of an indexing function. If $a \in A$ for $A$ an arena, an indexing function for $a$ is:

$$
\alpha:[a] \rightarrow \omega
$$

associating a copy index (a natural number) to each dependency of $a$, itself included. For $\alpha:[a] \rightarrow \omega$, we write $\operatorname{lbl} \alpha=a$ for its label and ind $\alpha=\alpha(\operatorname{lbl} \alpha)$ for its index, ie. the copy index it associates to the maximum of its domain.

Definition II.2. Let $A$ be an arena. There is an esp !A having:

- Events: indexing functions,

- Causality: $\alpha \leq \alpha^{\prime}$ defined as $\operatorname{lbl} \alpha \leq \operatorname{lbl} \alpha^{\prime}$ and $\alpha, \alpha^{\prime}$ agree on their common domain,

- Consistency: trivial - all finite sets consistent.

- Polarity: $\operatorname{pol}(\alpha)=\operatorname{pol}(\mathrm{lbl} \alpha)$.

Events $\alpha \in ! A$ inherit a Question/Answer labeling from A. We also define $!^{+} A$ duplicating only positive events, ie. with $\alpha$ : $[a] \rightarrow \omega$ such that for all $a^{\prime} \leq a$ with $\operatorname{pol}\left(a^{\prime}\right)=-, \alpha\left(a^{\prime}\right)=0$. 


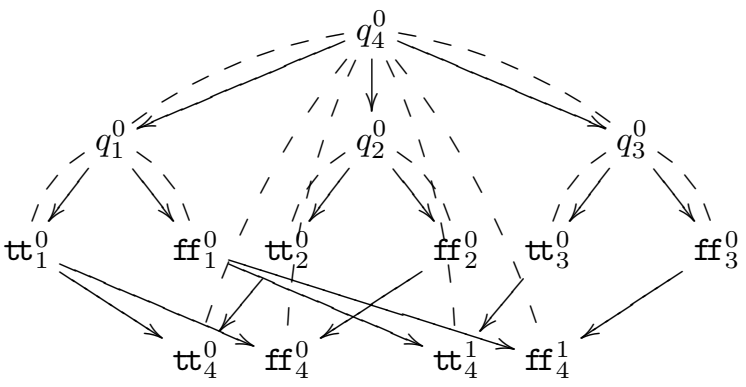

Fig. 2. The reduced pre-strategy for if : $\mathbb{B}_{1} \Rightarrow \mathbb{B}_{2} \Rightarrow \mathbb{B}_{3} \Rightarrow \mathbb{B}_{4}$

Configurations of $! A$ correspond to Boudes' thick subtrees [3] of arenas: a configuration $x \in \mathscr{C}(! A)$ visits a prefix of the arena $A$, but with branches duplicated at will. For now $! A$ is still technically an arena, but we will not consider it as such: in the compositional development to come we will equip it with an additional notion of symmetry (see Section III). Symmetry is key for the compositional structure of our model, but we can ignore it for the purposes of this section.

3) Pre-strategies: A map from event structure $A$ to event structure $B$ can be thought of as a simulation of $A$ within $B$. Formally it is a function $f: A \rightarrow B$ on events, which:

- Preserves configurations: for all $x \in \mathscr{C}(A), f x \in \mathscr{C}(B)$,

- Is locally injective: for all $x \in \mathscr{C}(A)$, for all $e, e^{\prime} \in x$, $f e=f e^{\prime} \Longrightarrow e=e^{\prime}$.

Additionally if $A$ and $B$ have polarity, $f$ preserves it.

Now, a pre-strategy on arena $A$ is defined as a map of esps.

$$
\sigma: S \rightarrow ! A
$$

This definition allows us to make formal the diagrams of the previous subsection: the nodes represent events of $S$, annotated with the label of the corresponding event in the game (as obtained through $\sigma$ ). The arrow $\rightarrow$ represents immediate causality in $S$, while the dashed lines - justification pointers - represent the relation induced on $S$ by immediate causality in $! A$. The diagrams of Subsection II-B were incomplete since they omitted copy indices. With these, the full (reduced - see below) pre-strategy for if is pictured in Figure 2.

The superscript of a move $s$ indicates its copy index, i.e. ind $(\sigma s)$. The full index function can be recovered from the copy index annotations of the dependencies in $! A$, as expressed by the dashed lines. As there are several compatible ways of returning tt (resp. ff), the pre-strategy has to use distinct copy indices for the projection to the game to be locally injective.

Any sequential innocent strategy on an arena $A$ in the sense of $\mathrm{HO}$ games [10] can be represented as a pre-strategy $\sigma$ : $S \rightarrow ! A$, where $S$ is the forest of correct P-views, and a $P$ view is sent by $\sigma$ to its latest move along with a copy index, chosen as to avoid collisions. However, there are many more pre-strategies, some non-deterministic or concurrent.

\section{Conditions for $\mathrm{PCF}_{\|}$-strategies}

Of course, not all pre-strategies $\sigma: S \rightarrow ! A$ are relevant for PCF. For the purposes of this paper, we need to describe a class of strategies containing our strategy for if and closed under composition (as will be made formal in the next section), but still small enough so that strategies within it have the same distinguishing power as terms of PCF.

A first remark is that $\mathrm{PCF}_{\|}$-strategies should be uniform, in the sense that their behaviour should not depend on Opponent's choice of copy indices. This seemingly elementary notion actually requires some machinery to formalize in a compositional setting. However, since in this section we only examine pre-strategies as purely static objects, we will content ourselves in examining reduced pre-strategies, which - as in the examples above - only acknowledge Opponent moves of copy index 0 , i.e. play on the expanded game $!^{+} A$. Uniformity aside, we now examine which properties $\mathrm{PCF}_{\|}$-strategies should satisfy w.r.t. causality and consistency. We introduce the conditions from the more robust to the more PCF-specific.

1) Courteous and receptive pre-strategies: Following [16] we define a strategy as a pre-strategy $\sigma: S \rightarrow !^{+} A$ which is:

- Receptive: For all $x \in \mathscr{C}(S)$, for all $\sigma x \subseteq^{-} y^{\prime}$, there is a unique $x \subseteq x^{\prime} \in \mathscr{C}(S)$ such that $\sigma x^{\prime}=y^{\prime}$.

- Courteous: For all $s_{1}, s_{2} \in S$ such that $s_{1} \rightarrow s_{2}$, if $\operatorname{pol}\left(s_{1}\right)=+$ or if $\operatorname{pol}\left(s_{2}\right)=-$ then $\sigma s_{1} \rightarrow \sigma s_{2}$.

In game semantics, receptivity is always present in one way or another. It is explicit and named contingent completeness in [10], but in most works on game semantics it is hardwired by asking that strategies contain only plays of even length (Opponent extensions being always present, they bring no additional information).

On the other hand, courtesy expresses that a strategy can only add causal links from negative to positive events. Of course it makes sense that if the rules of the game authorize Opponent to make a move, Player can not force them to wait. What might be more surprising is that Player is not capable of putting additional causal links between their own (positive) moves! One way to understand that is that strategies are thought of as interacting in a distributed fashion, over a network with an uncontrolled latency. So even though Player might want to play their moves in a specific order, they cannot control in what order they will reach their Opponent - this intuition is made formal by the result of [16] that receptive courteous strategies are exactly those that are unchanged by their composition with copycat.

Courtesy has a more chaotic history than receptivity: in essence, it is already present in interleaving-based game semantics for concurrency, where it forces strategies to be saturated under a number of permutations, in effect breaking unauthorized causal links. Under this form it dates back to [12], and is called saturation in [7] - a name that does not fit here, since it is not a saturation condition. In true concurrency approaches to game semantics, it appears under the name of "innocence" in [16, 6], and "courteousness" in Melliès and Mimram's asynchronous games [14]. We believe innocence is a slightly misleading name, since (as appears in this paper) it has no direct relationship with Hyland and Ong's notion of innocence, so we use courtesy instead. 
2) Visibility: In sequential HO games, strategy branches where Opponent always points to the previous move (ie. $P$ views) correspond to branches of terms. Here this intuition still holds, except that $P$-views are replaced by grounded causal chains. A grounded causal chain in $S$ is a sequence of events:

$$
\rho_{1} \rightarrow \ldots \rightarrow \rho_{n}
$$

where $\rho_{1} \in \min (S)$. If $\rho_{n}=s \in S$, we write $\rho \in \operatorname{gcc}(s)$; we also write $|\rho|=n$ for the length of $\rho$. In our games, grounded causal chains (gccs for short) give a notion of thread: if throws three sub-threads, and possibly merges some of them later. Each thread should go on independently, until it is merged with another or terminated. In particular, each thread should only use resources introduced within it; we call this visibility. A strategy $\sigma: S \rightarrow !^{+} A$ is visible iff:

$$
\forall s \in S, \forall \rho \in \operatorname{gcc}(s), \sigma \rho \in \mathscr{C}\left(!^{+} A\right)
$$

This amounts to $\rho$ containing all the justifiers (i.e. immediate dependencies in the game) of Player events. Since $\sigma$ is courteous, then in $\rho \in \operatorname{gcc}(s)$ causal links from positive to negative events match the causal links of the game - in other words Opponent points to the previous move, so the projection of $\rho$ to the arena $A$ is a $P$-view.

Visibility is of paramount importance in our development, and is indispensable to the stability under composition of almost all the forthcoming conditions. For visible strategies it makes sense to think of gccs as threads, and most of the remaining conditions restrict how strategies are allowed to generate, merge, or terminate threads.

3) Innocence: The sequential innocent deterministic strategies (described by a set of $P$-views) of [10] are the cornerstone of $\mathrm{HO}$ games. In our setting, they correspond to the (automatically visible) strategies $\sigma: S \rightarrow !^{+} A$ additionally satisfying:

(a) Backward sequentiality. The moves available to $\sigma$ are entirely determined by gccs: for all $s \in S,[s]$ is a gcc.

(b) Forward sequentiality. If $s \in S$ and $[s]$ extends by positive distinct $s_{1}, s_{2}$, then $[s] \cup\left\{s_{1}, s_{2}\right\} \notin \operatorname{Con}_{S}$.

(c) Determinism. Con $_{S}$ comprises all finite subsets of $S$.

It can be proved that strategies on $!^{+} A$ satisfying $(a),(b)$ and $(c)$ are in bijection with usual $\mathrm{HO}$ innocent strategies on $A$, up to the choice of copy indices. We note in passing that conditions (a) and (b) together are stable under our forthcoming notion of composition, yielding a notion of nondeterministic sequential innocence, a problem that is known to be difficult in sequential HO games.

However, for our purposes this definition does not fit: our parallel strategy for if fails both $(a)$ and $(b)$. We need to authorize prime configurations $[s]$ to be properly partially ordered, while ensuring that distinct Opponent moves following from the same player move are considered independently by Player. To formalize this, we say that for a strategy $\sigma: S \rightarrow !^{+} A$ a configuration $x \in \mathscr{C}(S)$ is normal if two Opponent moves never share the same justifiers: more formally, for all $s_{1}, s_{2} \in x$ negative events both minimal or such that there is $s \in S$ such that $s \rightarrow s_{1}, s \rightarrow s_{2}$ (or equivalently by courtesy, $\sigma s \rightarrow \sigma s_{i}$ ), we have $s_{1}=s_{2}$. Then, $\sigma$ is innocent iff its behaviour is specified by normal configurations: for all $s \in S,[s]$ is normal.

Concurrent innocence no longer implies visibility, although it will be stable under composition only in the presence of visibility. Finally, in the presence of forward sequentiality this definition is equivalent to the one above and so conservatively extends usual sequential innocent strategies.

4) Well-bracketing: We first introduce some notation and terminology. If $\sigma: S \rightarrow !^{+} A$ is a strategy, $S$ inherits a $\mathrm{Qu} / \mathrm{An}$ labelling from $A$ : an event $s \in S$ is a Question/Answer if $\mathrm{lbl}(\sigma s)$ is. We will sometimes annotate symbols for events with their $\mathrm{Qu} / \mathrm{An}$ or polarity labelling. We might say for instance "let $s^{-, \mathrm{Qu}} \in S$ ", meaning that $s$ is an Opponent Question. Given a set $X \in \operatorname{Con}_{S}$ and $s_{1}^{\mathrm{Qu}} \in X$, we say that $s_{1}$ is answered in $X$ if there is an answer $s_{2}^{\mathrm{An}} \in X$ with $\sigma s_{1} \rightarrow \sigma s_{2}$. Additionally $X$ is complete if all the questions of $X$ are answered in $X$. Finally If $\rho$ is a gcc in $S$, then we write $\rho_{i \leq \_} \leq j$ for the segment of $\rho$ lying between indices $i$ and $j$, endpoints included. We also use the strict variant $\rho_{i<_{-}<j}$. Finally, $\rho_{\omega}$ denotes the last event of a gcc $\rho$.

We now give conditions $(a),(b)$ and $(c)$ for well-bracketing. (a) In any gcc $\rho$ in $S$, if $\sigma \rho_{i} \rightarrow \sigma \rho_{j}^{\text {An }}$, then $\rho_{i}$ is the pending question (the latest unanswered question) in $\rho_{-}<j$, ie. gccs are well-bracketed in the usual sense. Lifting this condition would authorize strategies for control operators such as call/cc [11]. (b) Answering is affine: for $x \in \mathscr{C}(S)$ and two gccs $\rho, \rho^{\prime} \subseteq x$ such that $\rho_{\omega}$ and $\rho_{\omega}^{\prime}$ are both answers to the same question, then the greatest common event of $\rho \cap \rho^{\prime}$ is positive - so $\rho$ and $\rho^{\prime}$ fork at an Opponent event.

Not having condition $(b)$ means that besides having strategies for the booleans tt and ff one has also, among others, a strategy that answers both tt and ff: this strategy creates two copies of its runtime environment and throws two threads, returning $t t$ in the first and $f f$ in the second. There is a striking similarity with the $\mathrm{C}$ primitive fork, that creates two threads and gives each of them a unique identifier - the details of this connection are left for future work. Before we are done with well-bracketing, there is one last condition to mention:

(c) Take $\rho, \rho^{\prime} \in \operatorname{gcc}(s)$ for $s \in S$. Suppose moreover that $\rho_{i}=$ $\rho_{j}^{\prime}$ and that $\rho_{i<_{-}<|\rho|}$ and $\rho_{j<_{-}<\left|\rho^{\prime}\right|}^{\prime}$ are disjoint. Then $\rho_{i<_{-}<|\rho|}$ and $\rho_{j<\_<\left|\rho^{\prime}\right|}^{\prime}$ are complete. Graphically, in a diagram as below:

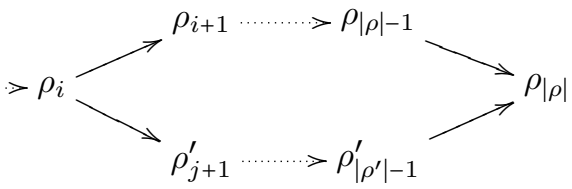

the two separate branches are complete - if Player creates two threads, he can only merge them again once all their questions are answered. A similar condition appears in Wall's games model for syntactic control of interference, see [17].

A visible strategy $\sigma: S \rightarrow !^{+} A$ is well-bracketed when it satisfies all three conditions $(a),(b)$ and $(c)$.

5) Decomposing $\mathrm{PCF}_{\|}$-strategies: As we shall see later, our interpretation of PCF will yield strategies satisfying these conditions. Note however that soundness for PCF depends on 
much weaker conditions (the key one is single-threadedness, see Subsection III-D). With the full set of conditions we aim for a much stronger correspondence with syntax; in fact we will describe in Subsection IV-2 a decomposition result akin to the usual definability of sequential innocent strategies.

\section{CONCURRENT HYLAND-ONG GAMES}

We now describe the mathematical structure required to compositionally generate the strategies described above from PCF terms. The basic ideas behind our mathematical development come from the games on event structures introduced in [16]. To express uniformity of strategies w.r.t. copy indices, we use the notion of symmetry on event structures [19]. In particular we will use a variant of the games on event structures with symmetry of [4].

\section{A. Event structures with symmetry}

A symmetry on an event structure (possibly with polarity)

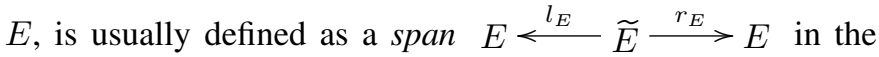
category of event structures (possibly with polarity), which is an equivalence relation in the categorical sense, $l_{E}$ and $r_{E}$ are rigid and are functional bisimulations, expressed as a configuration extension property (see [19]). Intuitively a symmetry on $E$ can be regarded as a proof-relevant notion of equivalence relation between configurations of $E$, in a way that respects the operations available on event structures.

In full generality, symmetry provides an abstract notion of bisimulation in a categorical setting [19]. In the concrete case of event structures, symmetries can be presented more concretely: any configuration $x \in \mathscr{C}(\widetilde{E})$ generates a bijection:

$$
\theta_{x}=\left\{\left(l_{E} \widetilde{e}, r_{E} \widetilde{e}\right) \mid \widetilde{e} \in x\right\}
$$

This $\widetilde{E}$ gives a collection of such isomorphisms. But actually symmetry on event structures can be axiomatized directly in terms of the isomorphisms generated. So although we will occasionally refer to symmetry presented as a span as above, we will often rely on the following equivalent presentation.

1) Isomorphism families: An isomorphism family on an event structure $E$ is a set of bijections $\theta: x \cong y$ where $x, y \in$ $\mathscr{C}(E)$. For $\theta$ in the isomorphism family, we write $x \stackrel{\theta}{\cong} \mathcal{E} y$. This set of bijections has to be closed under certain operations:

(1) Identity: for all $x \in \mathscr{C}(E), x \underset{\mathrm{id}_{x}}{\stackrel{\mathcal{E}}{E}}$.

(2) Inverse: if $x \stackrel{\theta}{\cong} \mathcal{E} y$ then $y \stackrel{\theta^{-1}}{\cong} \mathcal{E} x$.

(3) Composition: if $\underset{\theta}{\stackrel{\theta}{\cong} \mathcal{E}} y$ then $y \stackrel{\phi}{\cong} \mathcal{E} z$ then $x \stackrel{\phi \circ \theta}{\cong} \mathcal{E} z$.

(4) Restriction: if $\underset{\stackrel{\theta}{\cong}}{\cong} y$ and $x^{\prime} \subseteq x$ with $x^{\prime} \in \mathscr{C}(E)$, there is a (necessarily unique) $\theta^{\prime} \subseteq \theta$ and $y^{\prime} \in \mathscr{C}(E)$ such that $x^{\prime} \stackrel{\theta^{\prime}}{\cong} \mathcal{E} y^{\prime}$.

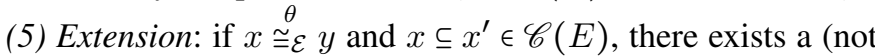
necessarily unique) $\theta \subseteq \theta^{\prime}$ and $y^{\prime} \in \mathscr{C}(E)$ such that $x^{\prime} \stackrel{\theta^{\prime}}{\cong} y^{\prime}$.

Conditions (1), (2) and (3) express that isomorphisms equip the set of configurations with a groupoid structure. Conditions (4) and (5) express that isomorphisms behave like a historypreserving bisimulation. The notion extends directly in the presence of other data such as polarity or Question/Answer labelling, which should be preserved by isomorphisms. An event structure with symmetry (resp. and polarity) is called an ess (resp. essp) for short. Event structures with a symmetry/an isomorphism family are denoted by $\mathcal{E}, \mathcal{F}, \mathcal{A}, \mathcal{B}, \ldots$

We will be particularly interested in essps with a unique choice of extensions for positive events: an essp $\mathcal{A}$ is thin iff for all $x \stackrel{\theta}{\cong} \mathcal{A} y$, if $\theta$ has positive extensions to valid isomorphisms $x_{1} \stackrel{\theta_{1}}{\cong} \mathcal{A} y_{1}$ and $x_{2} \stackrel{\theta_{2}}{\cong} \mathcal{A} y_{2}$, if $x_{1} \cup x_{2} \in \mathscr{C}(A)$ then $\theta_{1} \cup \theta_{2}$ is valid as well. In other words, the left projection of the symmetry reflects positive compatibility.

2) Maps and symmetry: For $\mathcal{E}$ and $\mathcal{F}$ ess and $f: E \rightarrow F$ a map between the underlying event structures, we can apply $f$ to bijections $\theta: x \cong y$ between configurations of $E$ using:

$$
f \theta=\left\{\left(f e_{1}, f e_{2}\right) \mid\left(e_{1}, e_{2}\right) \in \theta\right\}
$$

We say that $f: E \rightarrow F$ preserves symmetry (also written $f$ : $\mathcal{E} \rightarrow \mathcal{F}$ ) iff for all $\underset{\stackrel{\theta}{\cong}}{\cong} y, f \underset{\mathcal{f}}{\stackrel{\theta}{\cong}} \mathcal{F} f y$. There is a category $\mathcal{E S S}$ of ess and maps preserving symmetry, and a category $\mathcal{E S S P}$ in the presence of polarities. If $f, g: \mathcal{E} \rightarrow \mathcal{F}$ are parallel maps preserving symmetry we will say that they are symmetric, written $f \sim g$, whenever for all $x \in \mathscr{C}(E)$, we have:

$$
f x \stackrel{\{(f e, g e) \mid e \in x\}}{\cong} \mathcal{F}
$$

3) Games with symmetry: Instead of playing directly on arenas, our strategies will play on the derived games with symmetry. A thin concurrent game (tcg for short) is a tuple $(\mathcal{A}, \leq$, pol, $\lambda)$ satisfying the conditions of an arena except negativity, and equipped with an isomorphism family additionally assumed to be race-free: for all $x \stackrel{\theta}{\cong}_{A} y$ with a positive $\theta \cup\left\{\left(s_{1}^{+}, s_{2}^{+}\right)\right\}$and a negative extensions $\theta \cup\left\{\left(s_{3}^{-}, s_{4}^{-}\right)\right\}$ to valid isomorphisms, then $\theta \cup\left\{\left(s_{1}, s_{2}\right),\left(s_{3}, s_{4}\right)\right\}$ is in the isomorphism family as well. Moreover, we assume that $\mathcal{A}$ has two sub-essp $\mathcal{A}_{+}$and $\mathcal{A}_{-}$on events $A$, such that $\mathcal{A}_{+}$(resp. $\mathcal{A}_{-}^{\perp}$ ) is thin, and closed under all negative extensions of the isomorphisms in $\mathcal{A}$ (resp. $\mathcal{A}^{\perp}$ ). Anticipating the next subsection, this exactly means that the identity maps $\operatorname{id}_{A}: \mathcal{A}_{+} \rightarrow \mathcal{A}$ and $\operatorname{id}_{A}^{\perp}: \mathcal{A}_{-}^{\perp} \rightarrow \mathcal{A}^{\perp}$ are themselves $\sim$-strategies. We say that $\mathcal{A}$ and $\mathcal{A}^{\perp}$ have receptive thin sub-symmetries.

Essps support operations of simple parallel composition $\mathcal{A} \| \mathcal{B}$ (having as events the disjoint union $\{1\} \times A \cup\{2\} \times B$ and other components inherited) and dualization $\mathcal{A}^{\perp}$ (having polarity reversed, swapping $\mathcal{A}_{+}$and $\mathcal{A}_{-}$and leaving other components unchanged), see [4] for details - these operations preserve the conditions for tcgs.

If $A$ is an arena, we will be interested in particular in the following game with symmetry.

Definition III.1. From an arena $A$, the tcg $! A$ is defined as having components $(! A, \leq$, pol, $\lambda)$ as described in Subsection II-C, and isomorphism family comprising bijections $\theta: x \cong y$ such that $\mathrm{lbl} \circ \theta=\mathrm{lbl}$, and $\theta$ preserves and reflect causal order.

So, $\theta$ leaves invariant the label in the arena of the move played, and preserves justification - however, it can change copy indices. With this definition, $! A$ is a tcg: its negative 
and positive sub-essps respectively comprise isomorphisms preserving indices of positive (resp. negative) events.

\section{B. Uniform strategies}

We now define uniform strategies on tcgs, -strategies.

1) -pre-strategies: A -pre-strategy on a $\operatorname{tcg} \mathcal{A}$ is a map of essps:

$$
\sigma: \mathcal{S} \rightarrow \mathcal{A}
$$

so $S$ has to be equipped with a symmetry as well, preserved by $\sigma$. Our first condition on $\sim$-strategies is that they should behave as strategies in the sense of [16] at the level of symmetries. They should be receptive and courteous, but their action on symmetry should be receptive too: if $x \stackrel{\theta}{\cong} \mathcal{S} y$, if $\sigma \theta$ extends to

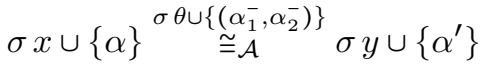

then there are uniquely defined $s, s^{\prime} \in S$ such that:

$$
x \cup\{s\} \stackrel{\theta \cup\left\{\left(s, s^{\prime}\right)\right\}}{\cong} \mathcal{S} \quad y \cup\left\{s^{\prime}\right\}
$$

with $\sigma s=\alpha$ and $\sigma s^{\prime}=\alpha^{\prime}$. We only have to require the existence, the uniqueness comes from receptivity of $\sigma$. A pre-strategy satisfying this is called strong-receptive.

2) Weak equivalence: Previously, we have seen that strategies with repetitions carry a choice of copy indices for their positive moves in ! $A$. In order to satisfy the laws of cartesian closed categories (ccc) required for soundness, we need to express that strategies play the same moves up to symmetry.

A weak equivalence between two $\sim$-pre-strategies $\sigma: \mathcal{S} \rightarrow$ $\mathcal{A}$ and $\tau: \mathcal{T} \rightarrow \mathcal{A}$ is given by two maps $f: \mathcal{S} \rightarrow \mathcal{T}$ and $g: \mathcal{T} \rightarrow \mathcal{S}$, making the two triangles commute up to symmetry:

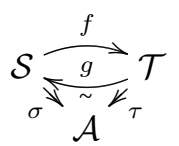

and such that $f \circ g \sim \operatorname{id}_{\mathcal{T}}$ and $g \circ f \sim \operatorname{id}_{\mathcal{S}}$. Weak equivalence is a canonical notion of equivalence between $\sim$-pre-strategies, as it expresses isomorphism up to symmetry. We write $\sigma \simeq \tau$ if $\sigma$ and $\tau$ are weakly equivalent, and $\sigma \cong \tau$ for the isomorphism - if all the symmetries involved are equalities.

3) -strategies: Unfortunately, the equivalence relation $\simeq$ fails to be a congruence on -pre-strategies: it is not preserved by composition (introduced in the next section). Proving that $\simeq$ is a congruence intuitively requires one to canonically transport configurations of strategies through isomorphisms in the game. As it is, symmetry ensures that such a transport exists, but not that it can be canonically chosen to induce a map. This is a very subtle point, which (among other reasons) motivated the introduction of saturated strategies in [4]. Saturated strategies are closed under the action of the symmetry on the game, hence providing a canonical way to transport configurations according to such isomorphisms.

Here, we follow a different route and require instead the symmetry of strategies to be as minimal as possible, formalized by the notion of thin essps introduced before. So formally, a -strategy on game with symmetry $\mathcal{A}$ is a courteous, strongreceptive and thin $\sim$-pre-strategy, meaning that its domain is thin. For an arena $A$ we can now define formally a $\mathrm{PCF}_{\|}$strategy $\sigma: \mathcal{S} \rightarrow ! A$ to be a $\sim$-strategy that is deterministic, innocent and well-bracketed.

The rest of this section is devoted to the construction of a ccc of $\sim$-strategies including $\mathrm{PCF}_{\|}$-strategies. First, we will organize tcgs and $\sim$-strategies into a compact closed category $\mathrm{Tcg} / \simeq$. Restricting on tcgs coming from arenas, we will form a cartesian closed sub-category $\mathrm{Cho/ \simeq} \mathrm{(for} \mathrm{"concurrent} \mathrm{Hyland-}$ Ong games") of $\mathrm{Tcg} / \simeq$, supporting the interpretation of $\mathrm{PCF}$.

In Section IV we will show that $\mathrm{PCF}_{\|}$-strategies form a sub-ccc of Cho/ $\simeq$, that is fully abstract for PCF.

\section{A category of $\sim$-strategies}

In this subsection, we will construct a -bicategory (a bicategory whose coherence laws hold up to $\sim$ only) Tcg (for thin concurrent games) having as objects tcgs, as morphisms $\sim$-strategies $\sigma: \mathcal{S} \rightarrow \mathcal{A}^{\perp} \| \mathcal{B}$ (also written $\sigma: \mathcal{A} \stackrel{\text { Tcg }}{\rightarrow} \mathcal{B}$, leaving $\mathcal{S}$ implicit), and weak equivalences as 2-cells. There is also a quotient category $\mathrm{Tcg} / \simeq$, however for now we refrain from quotienting: some of our later constructions will take place on concrete strategies rather than weak equivalence classes.

1) Pullbacks and parallel interaction: In game semantics, composition of strategies $\sigma: \mathcal{A} \stackrel{\text { Tcg }}{\rightarrow} \mathcal{B}$ and $\tau: \mathcal{B} \stackrel{\text { Tcg }}{\rightarrow} \mathcal{C}$ is usually performed by parallel interaction, where $\sigma$ and $\tau$ are allowed to communicate on $\mathcal{B}$, followed by hiding, where only the external events occurring on $\mathcal{A}$ or $\mathcal{C}$ are retained. Traditionally, parallel interaction is formulated as an intersection of the plays that both $\sigma$ and $\tau$ are prepared to play. In our games on event structures, parallel interaction is conveniently formulated as an adequate pullback. In general, the category of ess and maps preserving symmetry does not have all pullbacks, however it has enough of them:

Lemma III.2. For $\mathcal{A}$ a tcg and strong-receptive pre- strategies $\sigma: \mathcal{S} \rightarrow \mathcal{A}$ and $\tau: \mathcal{T} \rightarrow \mathcal{A}^{\perp}$, then temporarily forgetting about polarities there is a pullback in $\mathcal{E S S}$ :

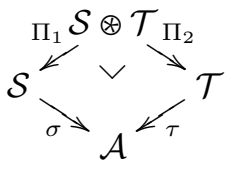

Arbitrary maps $f: \mathcal{A} \rightarrow \mathcal{C}$ and $g: \mathcal{B} \rightarrow \mathcal{C}$ might not have a pullback, because they might not agree on possible extensions of isomorphisms in $\mathcal{C}$, so the extension property might fail for the natural candidate for the isomorphism family of the pullback. However in the situation described in the lemma above, strong-receptivity of $\sigma$ and $\tau$ ensures that they will always find a common extension of a given isomorphism.

In this paper we will omit the construction of pullbacks, which can be found eg. in [20]. However we mention a key representation of their configurations and isomorphisms:

Proposition III.3. Let $\mathcal{A}$ be a tcg, and $\sigma: \mathcal{S} \rightarrow \mathcal{A}$ and $\tau: \mathcal{T} \rightarrow \mathcal{A}^{\perp}$ be $\sim$-strategies. Then the configurations of the 
(underlying event structure $S \otimes T$ of the) pullback $\mathcal{S} \otimes \mathcal{T}$ uniquely correspond to composite bijections:

$$
x \stackrel{\sigma}{\cong} \sigma x=\tau y \stackrel{\tau}{\cong} y
$$

with $x \in \mathscr{C}(S)$ and $y \in \mathscr{C}(T), x \stackrel{\sigma}{\cong} \sigma x$ is the bijection induced by $\sigma$, and the composite bijection is secured, ie. the transitive relation generated by $(s, t) \leq\left(s^{\prime}, t^{\prime}\right)$ if $s \leq s^{\prime}$ or $t \leq t^{\prime}$ is a partial order - which corresponds to the order of the pullback.

Isomorphisms of $\mathcal{S} \otimes \mathcal{T}$ correspond to commuting diagrams:

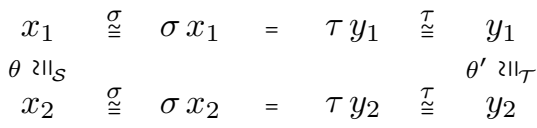

Interestingly, the symmetry $\overline{S \circledast T}$ is the pullback $\widetilde{S} \circledast \widetilde{T}$.

2) Projection and hiding: Now that we have a notion of parallel interaction, we need a way to express hiding. This is done via the notion of projection. If $E$ is an event structure and $V \subseteq E$ is a subset of the events of $E$, then there is a new event structure $E \downarrow V$ whose events are those in $V$, and whose causal order and conflict are inherited from $E$ [16].

If $\mathcal{E}$ is an ess, then $V \subseteq E$ is closed under symmetry when for all $v \in V$, for all $\underset{\stackrel{\theta}{\cong}}{\cong} y$ such that $v \in x$, we have $\theta v \in V$ as well. In that case, the set comprising bijections:

$$
x \cap V \stackrel{\theta \cap V^{2}}{\cong} y \cap V
$$

for all $x \stackrel{\theta}{\cong} \mathcal{E} y$ is an isomorphism family, generalizing the notion of projection in the presence of symmetry.

3) Composition: We finally define the composition of strategies. Take $\sigma: \mathcal{S} \rightarrow \mathcal{A}^{\perp} \| \mathcal{B}$ and $\tau: \mathcal{T} \rightarrow \mathcal{B}^{\perp} \| \mathcal{C}$ strongreceptive $\sim$-pre-strategies. We follow [16] and form:

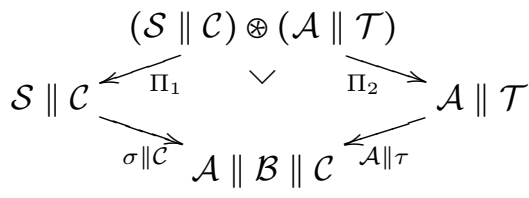

which exists by Lemma III.2. We write $\tau \otimes \sigma=(\sigma \| \mathcal{C}) \circ \Pi_{1}$. Then, we need to hide: we do that by forming

$$
V=\{v \in(S \| C) \otimes(A \| T) \mid \forall b \in B,(\tau \otimes \sigma) v \neq(2, b)\}
$$

so $V$ contains the events of the pullback that map either to $A$ or $C$, in other words which are external. The set $V$ is closed under symmetry, so we can form:

$$
\mathcal{T} \odot \mathcal{S}=(\mathcal{S} \| \mathcal{C}) \otimes(\mathcal{A} \| \mathcal{T}) \downarrow V
$$

then $\tau \otimes \sigma:(\mathcal{S} \| \mathcal{C}) \otimes(\mathcal{A} \| \mathcal{T}) \rightarrow \mathcal{A}\|\mathcal{B}\| \mathcal{C}$ restricts to

$$
\tau \odot \sigma: \mathcal{T} \odot \mathcal{S} \rightarrow \mathcal{A} \| \mathcal{C}
$$

and reinstating polarities, we obtain $\tau \odot \sigma: \mathcal{T} \odot \mathcal{S} \rightarrow \mathcal{A}^{\perp} \| \mathcal{C}$.

Interestingly, from this definition we have the isomorphism $\overline{T \odot S} \cong \widetilde{T} \odot \widetilde{S}$; in fact this could even be used as a definition of the symmetry of $T \odot S$.
4) Copycat: If $A$ is an esp, there is a new esp $C_{A}$ having:

- Events, those of $A^{\perp} \| A$,

- Causal order, the transitive closure of:

$$
\begin{aligned}
& \left\{\left((1, a),\left(1, a^{\prime}\right)\right) \mid a \leq a^{\prime}\right\} \cup\left\{\left((2, b),\left(2, b^{\prime}\right)\right) \mid b \leq b^{\prime}\right\} \\
\cup & \{((1, a),(2, a)) \mid \operatorname{pol}(a)=+\} \\
\cup & \{((2, a),(1, a)) \mid \operatorname{pol}(a)=-\}
\end{aligned}
$$

- Consistency, that of $A^{\perp} \| A$.

For $\mathcal{A}$ a game wih symmetry, we need to equip $\mathrm{CC}_{A}$ with a symmetry. There is a canonical choice: indeed as remarked in

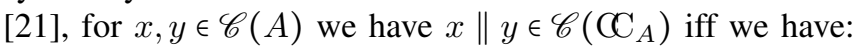

$$
x \supseteq^{+} \subseteq^{-} y
$$

Isomorphisms on $\mathrm{CC}_{A}$ are then defined as pairs of isomorphisms $x_{1} \stackrel{\theta}{\cong} \mathcal{A} x_{2}$ and $y_{1} \stackrel{\theta^{\prime}}{\cong} \mathcal{A} y_{2}$ such that the diagram

$$
\begin{array}{ccccc}
x_{1} & \beth^{+} & x_{1} \cap y_{1} & \subseteq^{-} & y_{1} \\
\theta<\|_{\mathcal{A}} & & \theta \cap \theta^{\prime} 2 \|_{\mathcal{A}} & & \theta^{\prime} 2 \|_{\mathcal{A}} \\
x_{2} & \beth^{+} & x_{2} \cap y_{2} & \subseteq^{-} & y_{2}
\end{array}
$$

commutes. Each such diagram induces a bijection between

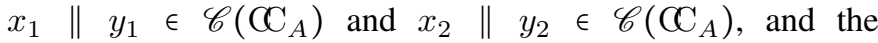
isomorphism family of $\mathrm{CC}_{\mathcal{A}}$ comprises all such bijections. For arbitrary essp $\mathcal{A}$, this need not be a valid isomorphism family as it can fail extension. However it is valid if the isomorphism family of $\mathcal{A}$ is race-free, which is the case for tcgs. In terms of symmetry, these definitions imply the isomorphism $\widetilde{\mathrm{CC}_{A}} \cong \mathrm{CC}_{\widetilde{A}}$.

Thus from a tcg $\mathcal{A}$ we obtain a $\sim$-pre-strategy $\boldsymbol{c}_{\mathcal{A}}$ : $\mathrm{CC}_{\mathcal{A}} \rightarrow \mathcal{A}^{\perp} \| \mathcal{A}$, which satisfies all the conditions to be a $\sim$-strategy. Our definitions of composition and copycat are compatible with those of [16], so for the same reason as there we know that there is a bicategory having arenas as objects, $\sim$-strategies as morphisms and isomorphisms preserving the projection to the game on the nose as 2-cells. However, we have argued before that we need to relate strategies via weak equivalences rather than isomorphisms, and the universal property of pullback does not ensure that weak equivalence is preserved under composition. Proving this, and setting up the framework so that it is true (while avoiding the saturation of [4]) was a significant part of the endeavour.

5) Bipullbacks and preservation of weak equivalence: Preservation of weak equivalence by composition relies on the observation that the pullbacks involved in the composition also satisfy a weaker universal property up to symmetry:

Lemma III.4. If $\sigma: \mathcal{S} \rightarrow \mathcal{A}$ and $\tau: \mathcal{T} \rightarrow \mathcal{A}^{\perp}$ are strongreceptive, courteous pre- -strategies with receptive thin subsymmetries, then their pullback:

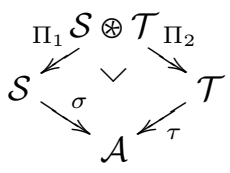

also satisfies the universal property of bipullbacks: for all $f: \mathcal{X} \rightarrow \mathcal{S}$ and $g: \mathcal{X} \rightarrow \mathcal{T}$ making the outer square commute 
up to symmetry (ie. $\sigma \circ f \sim \tau \circ g$ ), there exists $h: \mathcal{X} \rightarrow \mathcal{S} \otimes \mathcal{T}$, unique up to symmetry, such that $\Pi_{1} \circ h \sim f$ and $\Pi_{2} \circ h \sim g$.

The hypotheses of the lemma provide us, for each $x \in$ $\mathscr{C}(X)$, with configurations $f x \in \mathscr{C}(S)$ and $g x \in \mathscr{C}(T)$ and an isomorphism $\sigma(f x) \stackrel{\theta_{x}}{\cong} \mathcal{A} \tau(g x)$. Roughly, the proof of the lemma constructs interactively $y_{S}$ isomorphic to $f x$ and $y_{T}$ isomorphic to $g x$ such that $\sigma y_{S}=\tau y_{T}$, so that the pullback property can be applied. Each extension of $x$ is positive for either $S$ or $T$ : accordingly $S$ or $T$ has a canonical extension granted by its thin symmetry, to which the other strategy has to be receptive by strong-receptivity.

From this weaker universal property, it follows that weak equivalence is preserved by composition - note that applying this on the composition pullback uses the fact that tcgs have receptive thin sub-symmetries. The $\sim$-strategies are stable under composition, and comprise copycat. Similarly to [4], we have that Tcg is a -bicategory satisfying the laws of a compact closed category up to isomorphism and weak equivalence.

\section{A ccc of concurrent Hyland-Ong games}

We now describe a sub- -category Cho of Tcg, that gives a concurrent generalization of standard $\mathrm{HO}$ games.

Objects of Cho are negative arenas, ie. arenas whose minimal events are negative. A Cho-strategy from arena $A$ to arena $B$ (written $\sigma: A \stackrel{\text { Cho }}{\rightarrow} B$ ) is a negative $\sim$-strategy $\sigma: \mathcal{S} \rightarrow ! A^{\perp} \| ! B$ (ie. $S$ is negative), which is single-threaded:

(1) For any $s \in S,[s]$ has exactly one minimal event,

(2) For any $x \in \mathscr{C}(S)$ such that $x \cup\left\{s_{1}\right\}, x \cup\left\{s_{2}\right\} \in \mathscr{C}(S)$ and $x \cup\left\{s_{1}, s_{2}\right\} \notin \mathscr{C}(S)$, then $\left[s_{1}\right] \cap\left[s_{2}\right] \neq \varnothing$.

Both negativity and single-threadedness are stable under coitegativity ensures that $\sigma$ does not start playing on $A$, and single-threadedness will ensure that it satisfies surjective pairing. Finally, 2-cells are weak equivalences.

We mention a convenient operation for constructing strategies. Take a Cho-strategy $\sigma: \mathcal{S} \rightarrow ! B^{\perp} \| ! C$ and a strongreceptive, courteous map $f: ! B^{\perp} \rightarrow ! A^{\perp}$ Then, the composite:

$$
(f \| ! C) \circ \sigma: \mathcal{S} \rightarrow ! A^{\perp} \| ! C
$$

is still a Cho-strategy. Moreover if $A=B$ and $f \sim \mathrm{id}_{!} A$, it follows that the obtained map is weakly equivalent to $\sigma$.

1) Cartesian structure: Since morphisms in Cho are negative strategies and arenas are negative, it follows that the empty arena 1 is terminal. From two arenas $A$ and $B$, one can form the product arena $A \times B$; it is simply defined as $A \| B$. Firstly there are projections, obtained as:

$$
\varpi_{A}=\left(\mathrm{i}_{A} \| ! A\right) \circ \mathbb{C} ! A_{!}: \mathbb{C C}_{!_{A}} \rightarrow !(A \times B)^{\perp} \| ! A
$$

where $\mathrm{i}_{A}: ! A^{\perp} \rightarrow !(A \times B)^{\perp}$ is the injection map, which is strong-receptive (since $A, B$ are negative) and courteous. The other projection $\varpi_{B}: A \times B \stackrel{\text { Cho }}{\rightarrow} B$ is defined similarly.

From $\sigma: S \rightarrow ! A^{\perp} \| ! B$ and $\tau: T \rightarrow ! A^{\perp} \| ! C$, we define their pairing $\langle\sigma, \tau\rangle: A \stackrel{\text { Cho }}{\rightarrow \rightarrow} B \times C$. For that, we need to make sure that $\sigma$ and $\tau$ reach a disjoint image in $! A^{\perp}$. Take $A$ an arena, and $\iota: \omega \rightarrow \omega$ an injection. Then $\iota$ induces a map $\iota: ! A \rightarrow ! A$. The extended $\iota$ applies the injection to the copy indices of all minimal events, leaving the rest unchanged. More precisely, for $\alpha \in ! A, \iota(\alpha)$ is an index function $\alpha^{\prime}$ defined as:

$$
\begin{aligned}
& \alpha^{\prime}:[\operatorname{lbl} \alpha] \rightarrow \quad \omega \\
& a \mapsto \quad \mapsto \quad \iota(a)) \quad \text { if } a \in \min (A) \\
& a \quad \mapsto \quad \alpha(a) \quad \text { otherwise }
\end{aligned}
$$

Note that for any such $\iota$, the map $\iota^{\perp}: ! A^{\perp} \rightarrow ! A^{\perp}$ is strongreceptive and courteous, and we always have $\iota^{\perp} \sim \mathrm{id}_{!} A^{\perp}$, by definition of the symmetry on $! A$. Take two such injections that have a disjoint codomain, e.g. $\iota_{e}(n)=2 n$ and $\iota_{o}(n)=2 n+1$. Then, by the observation above we have Cho-strategies:

$$
\left(\iota_{e}^{\perp} \| ! B\right) \circ \sigma: A \stackrel{\text { Cho }}{\rightarrow} B \quad\left(\iota_{o}^{\perp} \| ! C\right) \circ \tau: A \stackrel{\text { Cho }}{\rightarrow} C
$$

with disjoint images in $! A^{\perp}$. So by co-pairing (and injection of $! B$ and $! C$ into $!(B \times C))$ we obtain:

$$
\langle\sigma, \tau\rangle: S\left\|T \rightarrow ! A^{\perp}\right\| !(B \times C)
$$

which is a Cho-strategy. It follows (by a reasoning similar to that for the neutrality of copycat) that $\varpi_{B} \odot\langle\sigma, \tau\rangle$ is isomorphic to $\left(\iota_{e}^{\perp} \| ! B\right) \circ \sigma$, which is weakly equivalent to $\sigma$ since $\iota_{e} \sim \operatorname{id}_{! A}-$ the same holds for $\varpi_{C} \odot\langle\sigma, \tau\rangle \simeq \tau$.

Finally, any $\sigma: A \stackrel{\text { Cho }}{\rightarrow} B \times C$ satisfies surjective pairing:

$$
\sigma \simeq\left\langle\varpi_{B} \odot \sigma, \varpi_{C} \odot \sigma\right\rangle
$$

This relies on the fact that Cho-strategies are single-threaded Indeed, for $\sigma: S \rightarrow ! A^{\perp} \| !(B \times C)$ single-threaded, $x \in \mathscr{C}(S)$ can be decomposed as $x=x_{B} \cup x_{C}$, where $x_{B}, x_{C} \in \mathscr{C}(S)$ are disjoint (by (1)) and only reach respectively $B$ and $C$. Likewise, two configurations $x_{B} \in \mathscr{C}(S), x_{C} \in \mathscr{C}(S)$ reaching only respectively $B$ and $C$ are compatible by (2) - the proof of the weak equivalence above follows these lines.

From all the above, it follows that Cho is cartesian up to weak equivalence. We now check that it is cartesian closed.

2) Exponentials: A morphism from $A \times B$ to $C$ in Cho is a map: $\sigma: S \rightarrow !(A \times B)^{\perp} \| ! C$. Up to isomorphism, its codomain is ! $A^{\perp} \| !\left(B^{\perp} \| C\right)$, which suggests $B^{\perp} \| C$ as an exponential object. However, $B^{\perp} \| C$ is not a valid arena, since it fails negativity - and negativity is essential to ensure that the empty arena 1 is a terminal object.

The standard arena construction $A \Rightarrow B$ introduced in Subsection II-C solves this issue by setting minimal events of $B$ as dependencies for events in $A$. Showing that this defines an exponential of $A$ and $B$ relies on the following lemma.

Lemma III.5. There is a bijection $\Phi$ preserving and reflecting weak equivalence between Cho-strategies $\sigma: C \stackrel{\text { Cho }}{\rightarrow} A \Rightarrow B$,

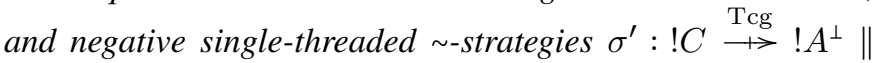
!B. Moreover, for all $\tau: D \stackrel{\text { Cho }}{\rightarrow} C, \Phi(\sigma \odot \tau) \simeq \Phi(\sigma) \odot \tau$.

Proof: First, we note that for arenas $A$ and $B$ there is a strong-receptive, courteous map of essp:

$$
\chi_{A, B}: !(A \Rightarrow B) \rightarrow ! A^{\perp} \| ! B
$$


Events $\beta:[(2, b)] \rightarrow \omega$ are kept unchanged. However, we have multiple copies of $A$ on the left hand side and only one on the right hand side, so we need to reindex minimal events of $A$ to avoid collisions. Therefore we send events $\alpha:[(1,(b, a))] \rightarrow \omega$ with $a$ minimal to $\left(1, \alpha^{\prime}\right)$ with $\alpha^{\prime}(a)=\langle\sharp b, \alpha((2, b)), \alpha((1,(b, a)))\rangle$ where $\sharp: B \rightarrow \omega$ is given by countability of $B$, and $\langle-,-,-\rangle: \omega^{3} \rightarrow \omega$ is any injection. If $a$ is non-minimal, its copy index is unchanged.

For $\sigma: \mathcal{S} \rightarrow ! A^{\perp} \| !(B \Rightarrow C), \Phi(\sigma)$ is $\left(! A^{\perp} \| \chi_{B, C}\right) \circ \sigma$. For $\left.\sigma: \mathcal{S} \rightarrow ! A^{\perp} \|\left(! B^{\perp} \| ! C\right)\right)$, by (1) of single-threadedness any $s \in S$ has a unique minimal dependency mapping to a minimal event of $! C$. Thus $\sigma$ factors uniquely through $! A^{\perp} \| \chi_{B, C}$ up to symmetry, from which the lemma follows.

From the lemma above, the cartesian structure (up to $\simeq$ ) of Cho and the compact closed structure (up to $\simeq$ ) of Tcg, it follows that Cho is cartesian closed (up to $\simeq$ ).

\section{E. Recursion}

Usually, the interpretation of the fixpoint combinator $Y$ is obtained by showing that the ccc of strategies is enriched over a category of sufficiently complete partial orders. Once this is established, the recursive equation for $Y$ can be solved by computing the right least upper bound. Here however (as in AJM games [1]) the ccc Cho/ $\simeq$ is a quotient, and it is not clear that the natural induced order on equivalence classes has the adequate completeness properties. Instead we use an ordering on concrete strategies rather than equivalence classes.

Definition III.6. Let $\sigma: \mathcal{S} \rightarrow \mathcal{A}$ and $\tau: \mathcal{T} \rightarrow \mathcal{A}$ be negative single-threaded $\sim$-strategies on a tcg $\mathcal{A}$. Then $\sigma \unlhd \tau$ iff we have the inclusion map $S \hookrightarrow T$ with all data in $\mathcal{S}$ coinciding with the restriction of that in $\mathcal{T}$, and for all $s \in S, \sigma s=\tau s$.

The $\sim$-strategies on $\mathcal{A}$ ordered by $\unlhd$ form a directed complete partial order (dcpo). It is not pointed though - it does not have a least element: indeed there is one minimal -strategy on $\mathcal{A}$ for each renaming of its minimal negative events. We call such a minimal -strategy empty. For each $\mathcal{A}$ we will consider one empty $\sim$-strategy in particular, written $\perp_{\mathcal{A}}: \min ^{-}(\mathcal{A}) \rightarrow \mathcal{A}$, that acts as the identity on the ess $\min ^{-}(\mathcal{A})$ having as events the minimal negative events of $\mathcal{A}$, with isomorphism family closed by receptivity. Not every $\sim$-strategy $\sigma: \mathcal{S} \rightarrow \mathcal{A}$ is above $\perp_{\mathcal{A}}$, however there is alway $\sigma^{\dagger}: \mathcal{S} \rightarrow \mathcal{A}$ such that $\sigma^{\dagger} \simeq \sigma$ and $\perp_{\mathcal{A}} \unlhd \sigma^{\dagger}$, obtained by renaming minimal events of $\mathcal{S}$.

Let us write $\mathcal{D}_{\mathcal{A}}$ for the pointed dcpo of $\sim$-strategies above $\perp_{\mathcal{A}}$. For an arena $A$ we can now define the following operation, using the ccc combinators of Cho:

$$
\begin{aligned}
F: \mathcal{D}_{!(A \rightarrow A)^{\perp} \| ! A} & \rightarrow \mathcal{D}_{!(A \rightarrow A)^{\perp} \| ! A} \\
\sigma & \mapsto\left(e v_{A, A} \odot\left\langle\mathbb{C}_{!(A \rightarrow A)}, \sigma\right\rangle\right)^{\dagger}
\end{aligned}
$$

Then $F$ is a continuous function, and has a least upper bound $Y=\vee F: A \Rightarrow A \stackrel{\text { Cho }}{\rightarrow} A$, which is easily shown using ccc laws to be a fixpoint combinator up to weak equivalence.

\section{F. Reduced form for $\mathrm{PCF}_{\|}$-strategies}

Describing Cho-strategies for basic combinators of PCF can be challenging, as one has to give the isomorphism family.
However, we show here that in the presence of visibility, innocence and determinism, full Cho-strategies can be recovered from the more compact (symmetry-free, Opponent replication free) notion of reduced $\mathrm{PCF}_{\|}$-strategies used in Section II - a situation analogous to the presentation of sequential innocent strategies as sets of P-views (compact, not compositional) or as sets of plays (not compact, compositional).

First of all, let us take a $\mathrm{PCF}_{\|}$-strategy $\sigma: \mathcal{S} \rightarrow ! A$. Its reduced form, written $\mathcal{S}_{\text {rf }}$, is the sub-event structure of $S$ comprising all events $s \in S$ such that for all $t^{-} \leq s$, ind $(\sigma t)=$ 0 . In other words, $\mathcal{S}_{\text {rf }}$ is the restriction of $S$ where Opponent moves always have a copy index of 0 - there is no Opponent duplication. The map $\sigma: \mathcal{S} \rightarrow ! A$ also induces a map of esp $\sigma_{\text {rf }}: \mathcal{S}_{\text {rf }} \rightarrow !^{+} A$. The event structure $\mathcal{S}_{\text {rf }}$ inherits a symmetry from $\mathcal{S}$; however then this symmetry is trivial. Indeed if $x, y \in$ $\mathscr{C}\left(\mathcal{S}_{\mathrm{rf}}\right)$ are such that $x \stackrel{\theta}{\cong} \mathcal{S} y$, then from $\sigma$ thin it follows that $x=y$ and $\theta=\mathrm{id}_{x}$. The symmetry on $\mathcal{S}_{\mathrm{rf}}$ is irrelevant, so $\sigma_{\mathrm{rf}}$ is a reduced $\mathrm{PCF}_{\|}$-strategy in the sense of Subsection II-D.

From reduced $\mathrm{PCF}_{\|}$-strategies we can build full strategies.

Lemma III.7. For a reduced $\mathrm{PCF}_{\|}$-strategy $\sigma: S \rightarrow !^{+} A$, there is a $\mathrm{PCF}_{\|}$-strategy $\bar{\sigma}: \bar{S} \rightarrow !$ A such that $\bar{\sigma}_{\mathrm{rf}} \simeq \sigma$.

Proof: The underlying event structure for $\bar{S}$ has for events indexing functions $\alpha:[s] \rightarrow \omega$ such that for all $t^{+} \leq s, \alpha(t)=$ 0 . Those are causally ordered as for $! A$, and all finite sets are consistent. The symmetry is designed as for $! A$.

The map $\bar{\sigma}$ sends $\left(\alpha^{-}:\left[s^{-}\right] \rightarrow \omega\right) \in \bar{S}$ to the event of ! $A$ with $\operatorname{lbl}(\sigma s)$ as label and ind $\alpha$ as copy index. For $\alpha^{+}$: $\left[s^{+}\right] \rightarrow \omega, \bar{\sigma} \alpha^{+}$has label $\operatorname{lbl}(\sigma s)$ and as index an injective function of the indices of $\sigma s$ and the negative dependencies of $s$. We get a $\mathrm{PCF}_{\|}$-strategy satisfying the required equation.

In fact, there is a bijection (up to weak equivalence) between $\mathrm{PCF}_{\|}$-strategies and reduced forms thereof. We define a $\mathrm{PCF}_{\|}$-strategy to be finite whenever its reduced form is.

Any $\mathrm{PCF}_{\|}$-strategy $\sigma: \mathcal{S} \rightarrow ! A$ is also a Cho-strategy: it is necessarily negative and is single-threaded by innocence.

\section{G. Interpretation of $\mathrm{PCF}$}

Any term $x_{1}: A_{1}, \ldots, x_{n}: A_{n} \vdash M: A$ of PCF is interpreted as a Cho-strategy $\llbracket M \rrbracket: \mathcal{S} \rightarrow ! \Pi_{1 \leq i \leq n} A_{i}{ }^{\perp} \| ! A$. The interpretation of $\mathrm{PCF}$ follows from the ccc structure, and from the strategies for the basic combinators of PCF described through their reduced forms in Figure 3 (op stands for succ or pred). In these diagrams we omit the copy index whenever it is 0. Missing from this figure is the reduced form of the strategy for if, which appears in Figure 2.

Theorem III.8. For all $\vdash M: \mathbb{X}, M \Downarrow$ iff $\llbracket M \rrbracket$ is non-empty.

Proof: From left to right (soundness), it follows from the ccc laws up to weak equivalence and the fact that the strategies of Figure 3 obey the required elementary equations. From right to left (adequacy), it follows from the same properties, with a straightforward use of logical relations. 


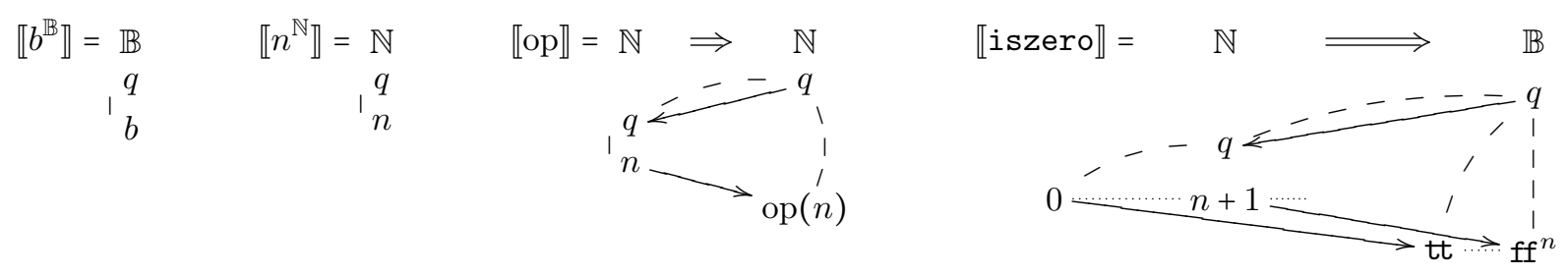

Fig. 3. Interpretation of the basic combinators of PCF

\section{FUll ABStRACTION FOR PCF}

We start this final section by the following proposition:

Proposition IV.1. There is a sub- -bicategory PcfPar of Cho with arenas as objects, negative $\mathrm{PCF}_{\|}$-strategies as morphisms, and weak equivalences as 2-cells. Moreover, PcfPar satisfies the laws of a ccc up to weak equivalence.

Proof: The difficult part is checking that composition preserves our conditions on strategies. Visibility and determinism are both stable under composition. Assuming visibility, so are innocence and well-bracketing.

Now, we prove that the extensional quotient of PcfPar is fully abstract for PCF. From now on we will often write $\sigma: \mathcal{A}$ instead of $\sigma: \mathcal{S} \rightarrow \mathcal{A}$ and leave $\mathcal{S}$ implicit.

We write $M \simeq$ obs $N$ for observational equivalence between terms, defined as usual [10]. We also define it on $\mathrm{PCF}_{\|}$ strategies by defining a test on arena $A$ as any negative $\mathrm{PCF}_{\|}$strategy $\alpha: ! A^{\perp} \| ! \mathbb{X}$. For $\sigma: ! A$ and $\sigma^{\prime}: ! A$ we say that $\sigma$ and $\sigma^{\prime}$ are observationally equivalent, written $\sigma \simeq$ obs $\sigma^{\prime}$, whenever for all $\alpha: ! A^{\perp} \| ! \mathbb{X}, \alpha \odot \sigma \simeq \alpha \odot \sigma^{\prime}$. For standard reasons, $\simeq_{\text {obs }}$ is preserved by all our constructions on strategies. To prove full abstraction, we prove a finite definability result up to $\simeq_{\text {obs }}$.

Take a type $A=A_{1} \rightarrow \cdots \rightarrow A_{n} \rightarrow \mathbb{X}$ of PCF, with $A_{i}=A_{i, 1} \rightarrow \cdots \rightarrow A_{i, p_{i}} \rightarrow \mathbb{X}_{i}$. An alternative presentation of the standard finite definability argument for PCF follows from the remark that any innocent $\sigma: A$ can be decomposed into a flow substrategy $\sigma_{\text {flow }}: \mathbb{X}_{1} \rightarrow \ldots \mathbb{X}_{n} \rightarrow \mathbb{X}$ (comprising the plays of $\sigma$ where Opponent only plays answers), and for each Player question $q$ in $\sigma_{\text {flow }}$ in $\mathbb{X}_{i}$, the data of argument sub-strategies $\sigma_{q, j}: A_{1} \rightarrow \cdots \rightarrow A_{n} \rightarrow A_{i, j}\left(1 \leq j \leq p_{i}\right)$ detailing Player's behaviour if Opponent was to interrogate the $j$-th argument of $A_{i}$. To PCF-define $\sigma$, it suffices to PCF-define $\sigma_{\text {flow }}$ and to inductively PCF-define the argument sub-strategies. Our finite definability process will follow these lines: we will first address the first-order case, and generalize to higher-order using a decomposition argument.

1) First-order case: We remark that on first-order types (ie. types of the form $\mathbb{X}_{1} \rightarrow \cdots \rightarrow \mathbb{X}_{n} \rightarrow \mathbb{X}$ ), $\mathrm{PCF}_{\|}$-strategies denote continuous functions between the corresponding Scott domains, and that the functions definable by $\mathrm{PCF}_{\|}$-strategies are exactly the PCF-definable functions (although they are not computed sequentially). We assume familiarity with the interpretation of PCF in Scott domains [15]. For a PCF type $A$, we write $\llbracket A \rrbracket_{\mathcal{D}}$ for the corresponding domain.

Firstly, any $\mathrm{PCF}_{\|}$-strategy on a ground type must be (by determinism and well-bracketing) either empty or weakly equivalent to the interpretation of a constant. We write $\uparrow \sigma \epsilon$ $\llbracket \mathbb{X} \rrbracket_{\mathcal{D}}$ for the corresponding domain element. Likewise any $d \in \llbracket \mathbb{X} \rrbracket_{\mathcal{D}}$ corresponds to a strategy $\downarrow d: \mathbb{X}$ defined by the interpretation of the corresponding PCF combinator. We generalise $\downarrow$ to first-order types by, for $\sigma: \Pi_{i \leq n} \mathbb{X}_{i} \stackrel{\text { PcfPar }}{\rightarrow} \mathbb{X}$ :

$$
\begin{aligned}
\downarrow \sigma: \Pi_{i \leq n} \llbracket \mathbb{X}_{i} \rrbracket_{\mathcal{D}} & \rightarrow \llbracket \mathbb{X} \rrbracket_{\mathcal{D}} \\
\left\langle x_{i} \mid i \leq n\right\rangle & \mapsto \downarrow\left(\sigma \odot\left\langle\uparrow x_{i} \mid i \leq n\right\rangle\right)
\end{aligned}
$$

The following lemma is a variant of the familiar "linear tests suffice" argument in innocent game semantics.

Lemma IV.2. For $\sigma, \tau: \Pi_{i \leq n} \mathbb{X}_{i} \stackrel{\text { PcfPar }}{\rightarrow} \mathbb{X}, \sigma \simeq$ obs $\tau$ iff $\downarrow \sigma=\downarrow \tau$.

A ground term is a term $x_{1}: \mathbb{X}_{1}, \ldots, x_{n}: \mathbb{X}_{n} \vdash M: \mathbb{X}$ of $\mathrm{PCF}$ extended with a constant $\perp_{\mathbb{X}}: \mathbb{X}$ for divergence, which contains no subterm of functional type. For a ground term $\Gamma \vdash$ $M: \mathbb{X}$, it is direct to prove by induction that $\downarrow \llbracket M \rrbracket=\llbracket M \rrbracket_{\mathcal{D}}$.

Finally, we prove finite definability on first-order types.

Lemma IV.3. If $\sigma: \Pi_{i \leq n} \mathbb{X}_{i} \stackrel{\text { PcfPar }}{\rightarrow} \mathbb{X}$ is finite negative, there is a ground $x_{1}: \mathbb{X}_{1}, \ldots, x_{n}: \mathbb{X}_{n} \vdash M_{\sigma}: \mathbb{X}$ s.t. $\llbracket M \rrbracket \simeq_{\text {obs }} \sigma$.

Proof: Exploiting determinism of $\sigma$, we construct by induction a ground term $M_{\sigma}$ performing the same queries as $\sigma$ in some sequential order, such that $\llbracket M_{\sigma} \rrbracket_{\mathcal{D}}=\downarrow \sigma$. By Lemmas IV. 2 and the remark above, it follows that $\llbracket M_{\sigma} \rrbracket \simeq_{\text {obs }} \sigma$.

2) Higher-order and full abstraction: Let $\sigma: \prod_{i \leq n} A_{i} \stackrel{\text { PcfPar }}{\longrightarrow}$ $\mathbb{X}$ be a finite $\mathrm{PCF}_{\|}$-strategy. We first extract:

$$
\mathcal{S}_{\text {flow }}=\left\{s \in S \mid \sigma([s]) \subseteq !\left(\Pi_{1 \leq i \leq n} \mathbb{X}_{i}\right)^{\perp} \| ! \mathbb{X}\right\}
$$

It inherits all the components of an ess from $\mathcal{S}$, and gives a negative $\mathrm{PCF}_{\|}$-strategy from $\Pi_{1 \leq i \leq n} \mathbb{X}_{i}$ to $\mathbb{X}$.

We call events $q^{(+, \mathrm{Qu})} \in \mathcal{S}_{\text {flow }}$ whose negative dependencies all have copy index 0 the primary questions of $S$. Each such $q$ maps to an initial question of some $A_{i}$, we write $Q_{i}$ for the set of primary questions mapping to $A_{i}$. From there, Opponent can ask for an arguments of type $A_{i, j}$ via an event $q_{q, j}^{k}$ (for all $k$ ) immediately dependent on $q$. We set:

$$
\mathcal{S}_{q, j}=\left\{s \in S \mid \exists k \in \omega, s \geq q_{q, j}^{k}\right\}
$$

It inherits an essp structure from $\mathcal{S}$. We reassign:

$$
\sigma_{q, j}: \mathcal{S}_{q, j} \rightarrow !\left(\Pi_{1 \leq i \leq n} A_{i}\right)^{\perp} \| ! A_{i, j}
$$

and check that $\sigma_{q, j}$ is a negative $\mathrm{PCF}_{\|}$-strategy - it is the $j$-th argument sub-strategy of $q$. 
We now reconstruct $\sigma$ from the flow and argument substrategies using the ccc combinators of PcfPar. We set:

$$
\sigma_{\text {flow }}: \mathcal{S}_{\text {flow }} \rightarrow !\left(\Pi_{1 \leq i \leq n} \Pi_{q \in Q_{i}} \mathbb{X}_{i}\right)^{\perp} \| ! \mathbb{X}
$$

The assignment $\sigma_{\text {flow }}$ follows canonically from $\sigma$ and the symmetry on $\mathcal{S}$, which to any Player question $q^{(+, \mathrm{Qu})} \in \mathcal{S}_{\text {flow }}$ depending on minimal event $q^{i+1}$, associates canonically some $q \in Q$. With this, we can finally state our decomposition result:

Proposition IV.4. For $\sigma: \Pi_{1 \leq i \leq n} A_{i} \stackrel{\text { PcfPar }}{+\rightarrow} \mathbb{X} a \mathrm{PCF}_{\|}$-strategy,

$$
\sigma \simeq \sigma_{\text {flow }} \odot\left\langle x_{i} \sigma_{q, 1} \ldots \sigma_{q, p_{i}} \mid 1 \leq i \leq n, q \in Q_{i}\right\rangle
$$

with $\sigma_{\text {flow }}$ and $\sigma_{q, j} \mathrm{PCF}_{\|}$-strategies. The notation refers to ccc combinators, with $x_{i}$ the $i$-th projection from $\Pi_{1 \leq i \leq n} A_{i}$.

The proof requires a careful analysis of the right-hand side composition, and relies on all the conditions of $\mathrm{PCF}_{\|}$strategies. From this immediately follows by induction:

Proposition IV.5. If $\sigma: \mathcal{S} \rightarrow ! \llbracket A \rrbracket$ is a finite $\mathrm{PCF}_{\|}$-strategy, there exists $\vdash M_{\sigma}: A$ such that $\llbracket M \rrbracket \simeq_{\mathrm{obs}} \sigma$.

From the above ingredients we obtain easily:

Theorem IV.6. $\mathrm{PcfPar} / \simeq_{\mathrm{obs}}$ is fully abstract for $\mathrm{PCF}$, ie. for all $\Gamma \vdash M: A, \Gamma \vdash N: A, M \simeq_{\mathrm{obs}} N$ iff $\llbracket M \rrbracket \simeq_{\mathrm{obs}} \llbracket N \rrbracket$.

Proof: A standard argument using soundness, adequacy, finite definability, and the easy fact that if two strategies can be distinguished by a test, then a finite test suffices.

\section{CONCLUSiON}

We described a ccc $\mathrm{Cho} / \simeq$ of concurrent non-deterministic strategies, which supports concurrent notions of visibility, well-bracketing and innocence that conservatively extends the usual ones. In this setting, we gave a parallel intensionally fully abstract interpretation of PCF.

As a first extension, we plan to give an intensional version of the fully abstract model of PCF extended with the concurrent primitive parallel-or [15]. This is trickier than it might seem: the concurrent strategy for parallel-or is non-deterministic, and linking its non-deterministic intensional behaviour to its deterministic extensional behaviour requires some care.

More generally, we plan to apply this framework to give fine-grained truly concurrent games model of higher-order effectful concurrent programming languages.

\section{ACKNOWLEDGMENT}

We gratefully acknowledge the support of the ERC Advanced Grant ECSYM. We also would like to thank Dan Ghica for suggesting the idea of a parallel model of PCF.

\section{REFERENCES}

[1] Samson Abramsky, Radha Jagadeesan, and Pasquale Malacaria. Full abstraction for PCF. Inf. Comput., 163(2):409-470, 2000.

[2] Samson Abramsky and Paul-André Melliès. Concurrent games and full completeness. In LICS, pages 431-442. IEEE Computer Society, 1999.
[3] Pierre Boudes. Thick subtrees, games and experiments. In Curien [5], pages 65-79.

[4] Simon Castellan, Pierre Clairambault, and Glynn Winskel. Symmetry in concurrent games. In Thomas A. Henzinger and Dale Miller, editors, CSL-LICS '14, Vienna, Austria, July 14 - 18, 2014, page 28. ACM, 2014.

[5] Pierre-Louis Curien, editor. TLCA 2009, Brasilia, Brazil, July 1-3, 2009. Proceedings, volume 5608 of LNCS. Springer, 2009.

[6] Claudia Faggian and Mauro Piccolo. Partial orders, event structures and linear strategies. In Curien [5], pages 95111.

[7] Dan R. Ghica and Andrzej S. Murawski. Angelic semantics of fine-grained concurrency. Ann. Pure Appl. Logic, 151(2-3):89-114, 2008.

[8] Tom Hirschowitz. Full abstraction for fair testing in CCS. In Reiko Heckel and Stefan Milius, editors, CALCO, volume 8089 of $L N C S$, pages 175-190. Springer, 2013.

[9] Tom Hirschowitz and Damien Pous. Innocent strategies as presheaves and interactive equivalences for CCS. Sci. Ann. Comp. Sci., 22(1):147-199, 2012.

[10] J. M. E. Hyland and C.-H. Luke Ong. On full abstraction for PCF: I, II, and III. Inf. Comput., 163(2):285-408, 2000.

[11] James Laird. Full abstraction for functional languages with control. In LICS, pages 58-67. IEEE Computer Society, 1997.

[12] James Laird. A game semantics of idealized CSP. Electr. Notes Theor. Comput. Sci., 45:232-257, 2001.

[13] Paul-André Melliès. Asynchronous games 4: A fully complete model of propositional linear logic. In LICS, pages 386-395. IEEE Computer Society, 2005.

[14] Paul-André Melliès and Samuel Mimram. Asynchronous games: Innocence without alternation. In Luís Caires and Vasco Thudichum Vasconcelos, editors, CONCUR, volume 4703 of $L N C S$, pages 395-411. Springer, 2007.

[15] Gordon D. Plotkin. LCF considered as a programming language. Theor. Comput. Sci., 5(3):223-255, 1977.

[16] Silvain Rideau and Glynn Winskel. Concurrent strategies. In $L I C S$, pages 409-418. IEEE Computer Society, 2011.

[17] Matthew Wall. Games for syntactic control of interference. PhD thesis, University of Sussex, 2004.

[18] Glynn Winskel. Event structures. In Wilfried Brauer, Wolfgang Reisig, and Grzegorz Rozenberg, editors, Advances in Petri Nets, volume 255 of LNCS, pages 325392. Springer, 1986.

[19] Glynn Winskel. Event structures with symmetry. Electr. Notes Theor. Comput. Sci., 172:611-652, 2007.

[20] Glynn Winskel. Event structures, stable families and games. Lecture notes, Aarhus University, At http://daimi.au.dk/ gwinskel, 2011.

[21] Glynn Winskel. Strategies as profunctors. In Frank Pfenning, editor, FoSSaCS, volume 7794 of $L N C S$, pages 418-433. Springer, 2013. 